\title{
The Neuroprotective Effects of Muscle-Derived Stem Cells via Brain-Derived Neurotrophic Factor in Spinal Cord Injury Model
}

\author{
Donghe Han, ${ }^{1}$ Shurui Chen, ${ }^{2}$ Shiqiang Fang, ${ }^{2}$ Shiqiong Liu, ${ }^{2}$ Meihua Jin, ${ }^{3}$ Zhanpeng Guo, \\ Yajiang Yuan, ${ }^{2}$ Yansong Wang, ${ }^{2}$ Chang Liu, ${ }^{4}$ and Xifan $\mathrm{Mei}^{2}$ \\ ${ }^{1}$ Department of Neurobiology, Jinzhou Medical University, Jinzhou, Liaoning Province 121000, China \\ ${ }^{2}$ Department of Orthopedics, The First Affiliated Hospital of Jinzhou Medical University, Jinzhou, Liaoning Province 121000, China \\ ${ }^{3}$ Department of Immunology, Jinzhou Medical University, Jinzhou, Liaoning Province 121000, China \\ ${ }^{4}$ Department of Endocrinology, The First Affiliated Hospital of Jinzhou Medical University, Jinzhou, Liaoning Province 121000, China
}

Correspondence should be addressed to Chang Liu; cliuxmei@outlook.com and Xifan Mei; xfmei@outlook.com

Received 22 January 2017; Revised 6 April 2017; Accepted 13 April 2017; Published 5 July 2017

Academic Editor: John H. Zhang

Copyright (C) 2017 Donghe Han et al. This is an open access article distributed under the Creative Commons Attribution License, which permits unrestricted use, distribution, and reproduction in any medium, provided the original work is properly cited.

\begin{abstract}
Muscle-derived stem cells (MDSCs) possess multipotent differentiation and self-renewal capacities; however, the effects and mechanism in neuron injury remain unclear. The aim of this study was to investigate the effects of MDSCs on neuron secondary injury, oxidative stress-induced apoptosis. An in vivo study showed the Basso, Beattie, and Bresnahan (BBB) score and number of neurons significantly increased after MDSCs' transplantation in spinal cord injury (SCI) rats. An in vitro study demonstrated that MDSCs attenuated neuron apoptosis, and the expression of antioxidants was upregulated as well as the ratio of Bcl-2 and Bax in the MNT (MDSCs cocultured with injured neurons) group compared with the NT (injured neurons) group. Both LC3II/LC3I and $\beta$-catenin were enhanced in the MNT group, while XAV939 (a $\beta$-catenin inhibitor) decreased the expression of nuclear erythroidrelated factor 2 (Nrf2) and LC3II/LC3I. Moreover, MDSCs became NSE- (neuron-specific enolase-) positive neuron-like cells with brain-derived neurotrophic factor (BDNF) treatment. The correlation analysis indicated that there was a significant relation between the level of BDNF and neuron injury. These findings suggest that MDSCs may protect the spinal cord from injury by inhibiting apoptosis and replacing injured neurons, and the increased BDNF and $\beta$-catenin could contribute to MDSCs' effects.
\end{abstract}

\section{Introduction}

Spinal cord injury (SCI) is one of the most devastating, traumatic conditions, accompanied by initial mechanical damage and secondary injuries [1]. The mechanical damage of nerve axons by a primary injury induces biochemical and cellular cascades. Therefore, transplanting stem cells is considered the most effective method to treat SCIs [2], and various types of cells have been used to regenerate and replace the injured neurons to improve functioning [3-5]. Despite ongoing efforts, stem cell grafting has not achieved the desired effects because of transplant rejection and donor cell demise [6]. Recently, accumulating data have suggested that secondary injures, such as oxidative stress and apoptosis, could impede the successful treatment of SCIs by causing irreversible damage to nerve tissues. In addition, the poor survivability of transplanted cells is due to secondary injury processes [7]. Combined with the fact that the incidence of traumatic spinal cord injury is increasing worldwide [8], this has prompted the search for more effective novel cells.

Muscle-derived stem cells (MDSCs) are a multipotent, somatic stem cell that can be easily obtained from skeletal muscle. MDSCs can be transplanted as an autologous graft and can be rapidly expanded in vitro to reach the clinically relevant numbers of cells. They are also advantageous because they are much more resistant to a toxic microenvironment such as hypoxia, attenuate fibrosis, and readily differentiate into muscle, bone, neural, endothelial, fibroblastic, and hematopoietic lineages $[9,10]$. Most importantly, MDSCs have an extended replicative lifetime [11]. However, the effects of MDSCs in neuron regeneration and proliferation are largely unknown. In the present study, we used SCI rats and 


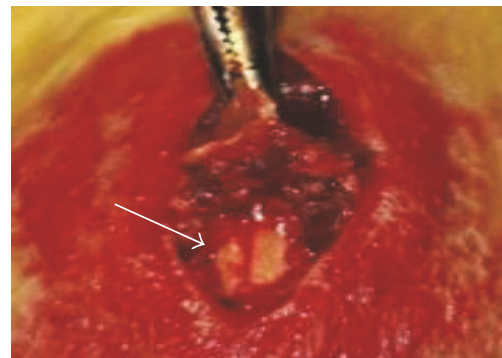

(a)

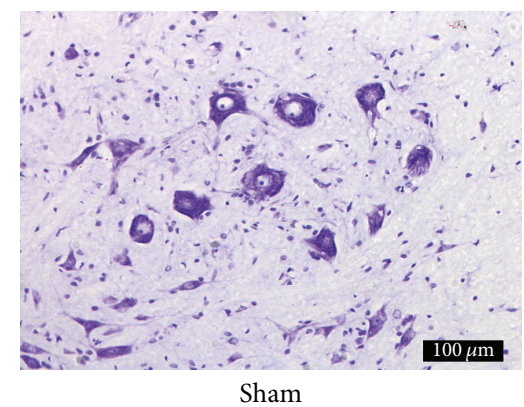

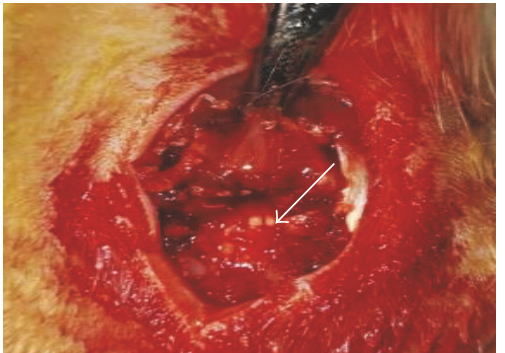

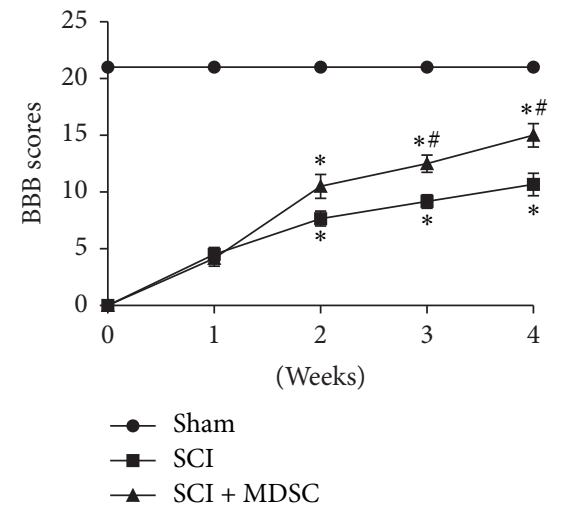

(c)

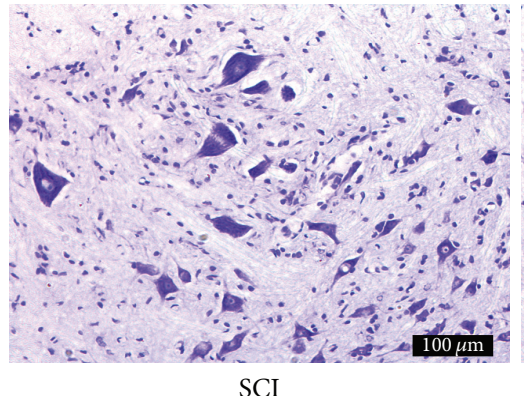

(d)

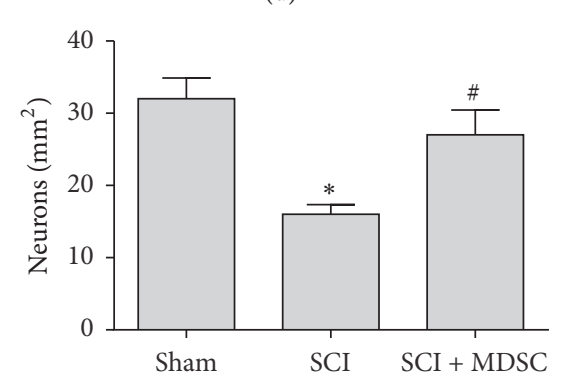

(e)

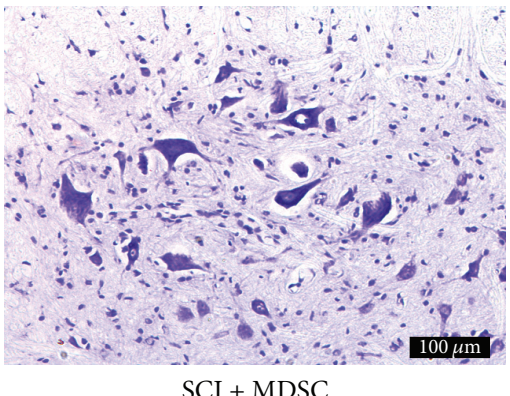

$\mathrm{SCI}+\mathrm{MDSC}$

FIGURE 1: Functional recovery after spinal cord injury: (a) exposed spinal cord (arrow shows intact spinal cord); (b) transected edges of spinal cord (arrow); (c) locomotor function was assessed by BBB test after spinal cord injury (SCI). (d) Nissl stain was performed 4 weeks after SCI. (e) Quantification of surviving neurons. Data was shown as mean $\pm \operatorname{SEM}(n=3)$; ${ }^{*} P<0.05$ versus sham. ${ }^{*} P<0.05$ versus SCI. Scale bar: $100 \mu \mathrm{m}$.

primary cultured neurons to investigate the effects of MDSCs on neuron injury after SCI.

\section{Materials and Methods}

2.1. SCI Model, Cell Transplantation, and Behavioral Test. Male adult SD rats weighing 200-220g were randomly divided into three groups of sham-operated $(n=6)$, SCI $(n=$ $6)$, and SCI + MDSC $(n=6)$. Laminectomies were carried out under anesthesia at the T9-T10 level (Figures 1(a) and 1(b)). One week after spinal cord injury, MDSCs (approximately $5 \times$ $10^{5}$ in $5 \mu$ l saline) were injected in the rostral and caudal of the lesion using a Hamilton syringe. An immunosuppressant and antibiotics were administered 1 day before transplantation and every day throughout the experiments. The locomotor function was assessed by the Basso, Beattie, and Bresnahan (BBB) locomotor rating scale once a week for 4 weeks. Four weeks after SCI, spinal cords were used for Nissl staining.

2.2. MDSC and Neuron Isolation. Newborn Sprague-Dawley rats were obtained from the Laboratory Animal Centre of Jinzhou Medical University (License umber SYXK (Liao) 2003-0011). The skeletal muscles from hind limbs were subjected to the isolation of MDSCs by preplating methods [12]. Muscles were cut into pieces, digested with a mixed enzyme solution (2.4\% type II dispersion enzyme, 1\% type II collagenase, and $2.5 \mathrm{mmol} / \mathrm{L} \mathrm{CaCl} 2$; Sigma, St. Louis, MO, USA) at $37^{\circ} \mathrm{C}$ for $45 \mathrm{~min}$., and filtered through a stainless-steel mesh 
with a pore diameter of $200 \mu \mathrm{m}$. The cells were centrifuged at $1,000 \mathrm{rpm}$ for $10 \mathrm{~min}$., and the pellets were resuspended in DMEM (Gibco, Santa Clara, CA, USA) with 10\% fetal calf serum (Gibco), 10\% horse serum, and 0.5\% chick embryo extract (US Biological, Marblehead, MA, USA). Cells were cultured in $25 \mathrm{~mL}$ culture flasks for 2 hours (recorded as preplate1 or $\mathrm{pP} 1$ ) and then centrifuged at $1,000 \mathrm{rpm}$ for 10 min., followed by daily transfers of nonadherent cells and replatings for 5 days until preplating 6 (pP5). All animal experiments were conducted in accordance with the National Institute of Health Guide for the Care and Use of Laboratory Animals.

The brain cortex was isolated from the same rats and gently dissociated to release the neurons. The cell suspensions were plated at a density of $1 \times 10^{6} / \mathrm{mL}$ with a Neurobasal Medium (Gibco) supplemented with $1 \mathrm{mM}$ glutamine (Amresco, OH, USA), $100 \mathrm{U} / \mathrm{mL}$ penicillin/streptomycin, and B27 (Gibco). The cells were grown in a humidified incubator maintained at $37^{\circ} \mathrm{C}$ with $5 \% \mathrm{CO}_{2}$.

2.3. The Coculture System and Grouping. To mimic injured conditions of spinal cord injury in vitro, primary neurons isolated from the brain (NC) were cultured with tert-butyl hydroperoxide (t-BHP, $200 \mu \mathrm{mol} / \mathrm{L}$ ) (Sigma), for $4 \mathrm{~h}$. Then the injured neurons $\left(5 \times 10^{6}\right.$ cells/well $)$ were cultivated without (NT group) or with (MNT group) MDSCs $\left(1 \times 10^{6}\right.$ cells/well) or treated with XAV939 ( $8 \mu \mathrm{M}, \mathrm{MNT}+\mathrm{XAV939})$ (MedChem Express, NJ, USA). The supernatant was used to measure BDNF, and injured neurons were subjected to staining and apoptosis.

To confirm the effects of brain-derived neurotrophic factor (BDNF) on the MDSCs, we set up an induced group in which MDSCs were cultured with $20 \mathrm{ng} / \mathrm{mL}$ BDNF (B3795, Sigma, USA) and an MDSC group without BDNF as a control. The morphological changes of the cells were observed under a phase-contrast microscope, and neuron-specific enolase (NSE) staining was performed after 6 days.

2.4. Nuclear Staining for Apoptotic Neurons. Cell climbing sheets on the second, fourth, and sixth days were fixed in 4\% paraformaldehyde. After staining with Hoechst 33258 ( $5 \mu \mathrm{g} / \mathrm{mL}$; 94403, Sigma), the cells were observed under an inverted fluorescent microscope. Five fields were randomly chosen, and the apoptotic cells were counted.

2.5. Flow Cytometry Analysis for t-BHP-Induced Apoptosis. To quantify cell apoptosis, an annexin V-fluorescein isothiocyanate (V-FITC)/propidium iodide (PI) (sc-4252 AK, Santa Cruz Biotechnology, Santa Cruz, CA, USA) double staining assay was performed per the manufacturer's instructions.

2.6. RNA Extraction and qPCR. After 6 days' culture, neurons were collected, and total RNA was isolated using a TRIzol reagent; $5 \mu \mathrm{g}$ of each sample was used to produce cDNA. Reverse transcription and amplification of target genes were performed according to the manufacturer's instructions (11752 and FERK0241, Invitrogen, CA, USA). The PCR reactions were carried out at $95^{\circ} \mathrm{C}$ for $2 \mathrm{~min}$., followed by 40 cycles of
TABLE 1: Primers used for the gene expression analysis.

\begin{tabular}{ll}
\hline Genes $^{\mathrm{a}}$ & Sequences $^{\mathrm{b}}\left(5^{\prime}-3^{\prime}\right)$ \\
\hline Bcl-2 & F: CTGGTGGACAACATCGCTCTG \\
& R: GGTCTGCTGACCTCACTTGTG \\
Bax & F: GCGAATTGGAGATGAACTGG \\
& R: GTGAGCGAGGCGGTGAGGAC \\
FO-1 & F: GCCTGCTAGCCTG GTTCAAG \\
& R: AGCGGTGTCTGGGATGAACTA \\
GCLC & F: GTCCTCAGGTGACATTCCAAGC \\
& R: TGTTCTTCAGGGGCTCCAGTC \\
NQO1 & F: GGCAGAAGAGCACTGATCGTA \\
& R: TGATGGGATTGAAGTTCATGGC \\
GAPDH & F: AAGCTGGTCTCAACGGGAAAC \\
& R: GAAGACGCCAGTAGACTCCACG \\
\hline
\end{tabular}

Bcl-2: B-cell lymphoma 2; Bax: Bcl-2-like protein 4; Nrf2: nuclear erythroidrelated factor 2; HO-1: heme oxygenasel; GCLC: glutamate-cysteine ligase catalytic subunit; NQO1: NAD(P) H: quinine oxidoreductase-1.

$95^{\circ} \mathrm{C}$ for $15 \mathrm{~s} ., 60^{\circ} \mathrm{C}$ for $30 \mathrm{~s}$., and $72^{\circ} \mathrm{C}$ for $30 \mathrm{~s}$. with a final extension at $72^{\circ} \mathrm{C}$ for $5 \mathrm{~min}$. GAPDH was used as an internal control. The primer sequences are shown in Table 1.

2.7. Western Blot. After 6 days' culture, neurons were collected and homogenized in RIPA buffer (Tris- $\mathrm{HCl}, \mathrm{pH} 7.4$ ), $150 \mathrm{NaCl}, 2$ EDTA, 1\% IGEPAL, 0.1\% SDS, protease inhibitor cocktail, and phosphatase inhibitor. Subsequently, $30 \mu \mathrm{g}$ of protein was separated by PAGE gel and transferred to a PVDF membrane. After blocking in Tris buffer saline-1\% nonfat dry milk for $1 \mathrm{~h}$., membranes were probed with primary antibody Nrf2 (1:1,000, ab31163), LC3 (1:1,000, ab48394), and $\beta$-catenin $\left(1: 1,000\right.$, ab32572) overnight at $4^{\circ} \mathrm{C}$. HRPconjugated secondary antibodies were used for the detection of primary antibodies (1:10,000, ab6721). GAPDH $(1: 2,000$, ab9485) was used as a control in the same sample. Signals were visualized using the ECL kit (34080, Thermo Fisher Scientific, USA). A densitometry analysis was conducted using ImageJ software (NIH, Bethesda, MD). All experiments were repeated at least 3 times $(n=3)$ using independent samples. All antibodies were purchased from Abcam (Abcam, Cambridge, MA, USA).

2.8. BDNF-Release Assay. To measure BDNF levels, the cellcultured supernatants (Day 2, Day 4, and Day 6) were subjected to an enzyme-linked immunosorbent assay (EK0307RB, Wuhan Boster, China). The assay was performed according to the manufacturer's protocol.

2.9. Immunocytochemistry. The MDSCs were fixed with an ice-cold $4 \%$ paraformaldehyde, incubated with $3 \% \mathrm{H} 2 \mathrm{O} 2$ for 30 min., and blocked with goat serum. The cells were then incubated with neuron-specific enolase (NSE) (1:100, sc51880 ) overnight at $4^{\circ} \mathrm{C}$, followed by a goat anti-mouse IgG (1:100; sc-2005, Santa Cruz Biotechnology, USA). Next, cells were incubated with DAB solution (D3939, Sigma, USA). 
TABLE 2: Strength of the correlation.

\begin{tabular}{lc}
\hline Absolute value of $R$ & Qualitative description of the strength \\
\hline $0.8-1$ & Very strong \\
$0.6-0.79$ & Strong \\
$0.40-0.59$ & Moderate \\
$0.2-0.39$ & Week \\
$0-0.19$ & Very week \\
\hline
\end{tabular}

2.10. Statistical Analysis. The results are presented as the mean \pm SEM. Analysis of variance (ANOVA) was performed to determine the differences among the groups. Post hoc tests were conducted using Bonferroni comparisons. A Pearson correlation analysis was performed to investigate the relation between the number of apoptotic cells and expression of BDNF. Table 2 provides a good indication of the qualitative description of the strength of the linear relationship and the absolute value of $R$. A $P$ value $<0.05$ was considered statistically significant.

\section{Results}

3.1. Effects of MDSC on Spinal Cord-Injured Rats. To examine the effects of MDSCs on spinal cord injury, locomotor function recovery after SCI was assessed with the BBB test, and there was no significant difference between the two SCI groups before MDSC transplantation. Prior to treatment, the mean BBB Locomotor Scores of all SCI rats were significantly decreased. In the first week posttreatment, a significantly faster functional recovery was observed in the SCI + MDSC group. The improvement in locomotor behavior was significant and could be observed until the end of the experiment (Figure 1(c)).

To determine the effects of MDSC on spinal cord tissue, a Nissl staining was performed. The number of surviving neurons was lower in the SCI group than in the sham group $(P=0.002)$; however, the number of surviving neurons increased more in the SCI + MDSC group than the SCI group $(P=0.034)$. These results demonstrate the significant protective effect of MDSCs (Figures 1(d) and 1(e)).

3.2. Effects of MDSCs on Neuron Apoptosis. As shown in Figure 2, the ratio of cell apoptosis of the NT group gradually increased with time. The apoptosis rate of the MNT group also showed an increase; however, that of the MNT group was significantly lower than the NT group (Figures 2(a) and 2(b)).

The flow cytometric analysis showed that there were far fewer apoptotic cells in the MNT group than in the NT group at the same time point, although the number of apoptotic cells in the MNT group was still higher than in the NC group (Figure 2(c)).

3.3. Effects of MDSCs on Oxidative Stress-Induced Apoptosis. Compared to the NC group, the gene expression of Bcl2 decreased in the NT group and MNT group $\left(P_{\mathrm{NT}}=\right.$ $\left.0.0001, P_{\mathrm{MNT}}=0.0001\right)$, while the expressions of the Bax in both groups were increased $\left(P_{\mathrm{NT}}=0.0001, P_{\mathrm{MNT}}=0.0081\right)$.
There was, however, more Bax gene expression in the NT group than in the MNT group ( $P=0.0028)$, while the expression of $\mathrm{Bcl}-2$ was less in the NT group than in the MNT group ( $P=0.0079)$, and the ratio between $\mathrm{Bcl}-2$ and Bax in the MNT group was higher than in the NT group $(P=0.0005)$ (Figures 3(a) and 3(b)).

The expression of an antioxidant, Nrf2, and its target genes, HO-1, GCLC, and NQO1, decreased in the NT group $\left(P_{\mathrm{Nr} 2}=0.0154, P_{\mathrm{HO}-1}=0.0126, P_{\mathrm{GCLC}}=0.0187, P_{\mathrm{NQO}-1}=\right.$ $0.0204)$, while those in the MNT group were enhanced compared with the NC group $\left(P_{\mathrm{Nrf} 2}=0.0001, P_{\mathrm{HO}-1}=\right.$ $0.0001, P_{\mathrm{GCLC}}=0.0001$, and $\left.P_{\mathrm{NQO}}=0.0033\right)$. The ratio of LC3II and LC3I was also increased in both NT and MNT groups $\left(P_{\mathrm{NT}}=0.0015, P_{\mathrm{MNT}}=0.0001\right)$. On the contrary, the levels of aforementioned proteins and genes in the MNT group were significantly high in comparison to the NT group $\left(P_{\mathrm{Nrf} 2}=0.0001, P_{\mathrm{HO}-1}=0.0001, P_{\mathrm{GCLC}}=0.0001, P_{\mathrm{NQO}-1}=\right.$ 0.0001 , and $\left.P_{\text {LC3II/LC3I }}=0.0001\right)($ Figures $3(\mathrm{c})-3(\mathrm{e}))$.

3.4. Effects of MDSCs on BDNF. To investigate the effects of MDSCs on growth factors, the expression of BDNF in supernatant was examined. The level of BDNF at day 6 in the NT group and MNT group decreased relative to the NC group $\left(P_{\mathrm{NT}}=0.0001, P_{\mathrm{MNT}}=0.0122\right)$. However, the level of BDNF was much higher in the MNT group than in the NT group $(P=0.0024)$ (Figure 3(f)).

3.5. Effects of MDSCs on $\beta$-Catenin. To investigate the mechanism of MDSCs, the expression of $\beta$-catenin was examined. As Figure 3 shows, $\beta$-catenin decreased in the NT group $(P=0.0401)$, while that in the MNT group increased compared with the NT group $(P=0.0001)$ (Figures $3(\mathrm{c})$ and $3(\mathrm{~d}))$. In addition, increased LC3II/LC3I and Nrf2 in the MNT group were suppressed with XAV939 $(\beta$-catenin inhibitor $)$ treatment $\left(P_{\mathrm{LC} 3 \mathrm{II} / \mathrm{LC} 3 \mathrm{I}}=0.0001, P_{\mathrm{Nrf} 2}=0.0001\right)$ (Figures 4(a) and 4(b)). Compared to the MNT group, the expression of Bax was upregulated, while the ratio of $\mathrm{Bcl} 2 / \mathrm{Bax}$ was downregulated when adding XAV939 to the MNT group $\left(P_{\mathrm{Bax}}=0.0309, P_{\mathrm{Bcl} 2 / \mathrm{Bax}}=0.028\right)$ (Figures $4(\mathrm{c})$ and $\left.4(\mathrm{~d})\right)$.

3.6. Morphological Changes of MDSCs. To further verify the effects of BDNF on MDSCs' differentiation under the toxic microenvironment, we performed an in vitro study using primary neurons. The MDSCs of the MNT group showed morphological changes from the fourth day. The cells began to shrink and extend neurites (Figure 5(a)), which connected with one another as time progressed. To verify whether they had neuronal characteristics, the cells were stained with NSE, and it showed positive results for the MDSCs in the cocultured group (Figure 5(b)). In the experiment to test the effects of exogenous BDNF on the MDSCs, it is evident that the cytoskeleton retracted more progressively to the cell centre, and the extended neurites also connected with one another, which showed NSE-positive results (Figures 5(c) and $5(\mathrm{~d})$ ). The cells in the control group (MDSCs without BDNF) showed no morphological changes, and the NSE staining results were negative (Figures 5(e) and 5(f)). 

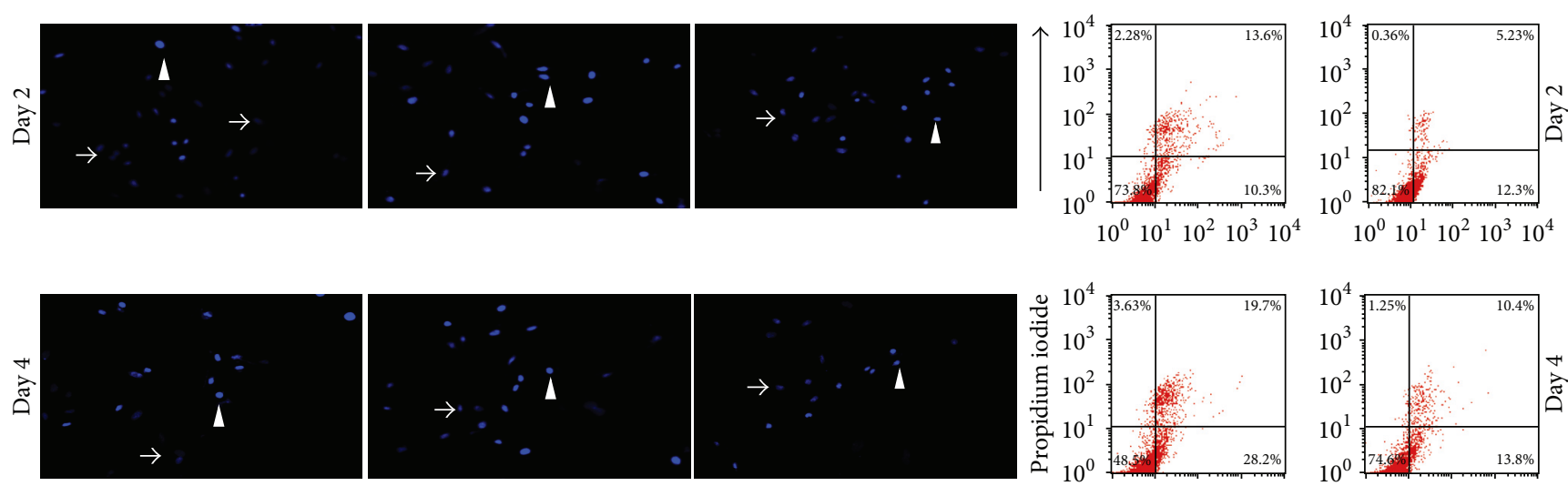

$10^{0} 10^{1} 10^{2} 10^{3} 10^{4}$

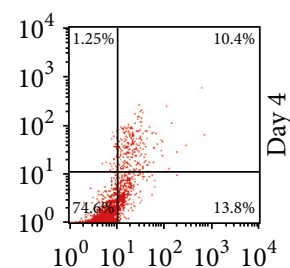

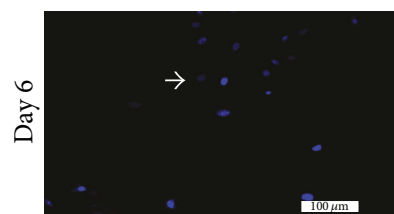

NC

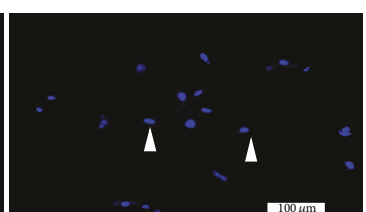

NT

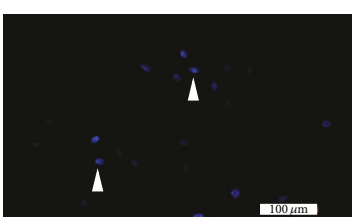

MNT

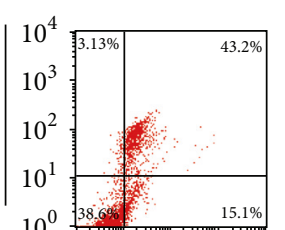

$10^{0} 10^{1} 10^{2} 10^{3} 10^{4}$

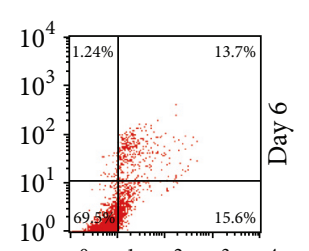

$10^{0} 10^{1} 10^{2} 10^{3} 10^{4}$ MNT

(a)

(c)

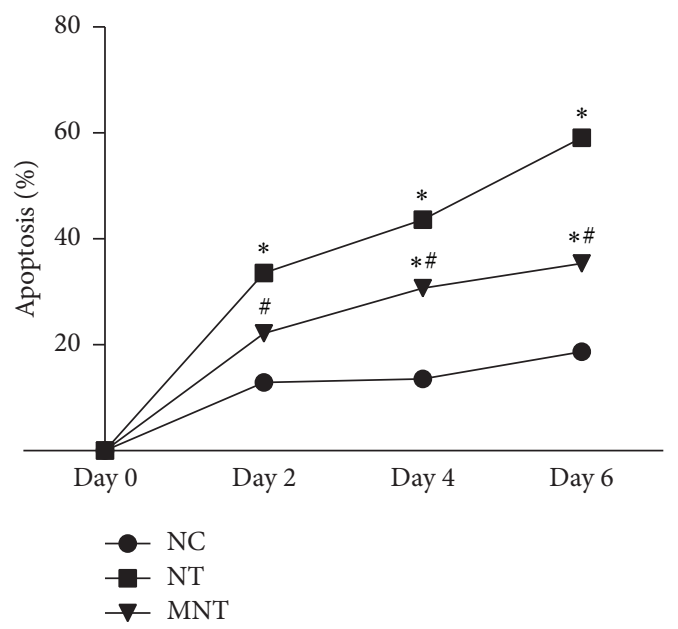

(b)

FIGURE 2: Effects of MDSCs on neurons. (a) The neurons stained with Hoechst 33258. Arrowheads (ム) indicate normal neurons and arrows $(\uparrow)$ indicate apoptotic neurons. Five fields were randomly selected and the (b) apoptotic rates were measured. (c) The ratio of apoptosis of neurons was measured by Annexin V-FITC/propidium iodide double staining. Data was shown as mean \pm SEM $(n=5)$, ${ }^{*} P<0.05$ versus NC group, and ${ }^{\#} P<0.05$ versus NT group. NC (no treatment); NT (neurons treated with t-BHP); MNT (neurons cocultured with MDSCs after treatment with t-BHP). Scale bar: $100 \mu \mathrm{m}$.

3.7. The Relationship between Apoptosis and BDNF. The secretion of BDNF in the NT and MNT groups was gradually decreased from day 2, while the levels of BDNF in MNT groups were much higher than those in NT groups at the same time point $(P<0.001)$ (Figure $5(\mathrm{~g}))$. Numbers of apoptotic cells in both coculture groups were gradually upregulated compared to the NC group. However, those in the MNT group were much lower compared with the NT group $(P<$ 0.001 ) (Figure 5(h)).

To investigate the correlation between the number of apoptotic cells and levels of BDNF, a linear regression analysis was performed. Results showed a strong negative correlation regarding the number of apoptotic cells and the expression of $\operatorname{BDNF}(R=-0.775, P<0.0001$, Figure $5(i))$, suggesting the 

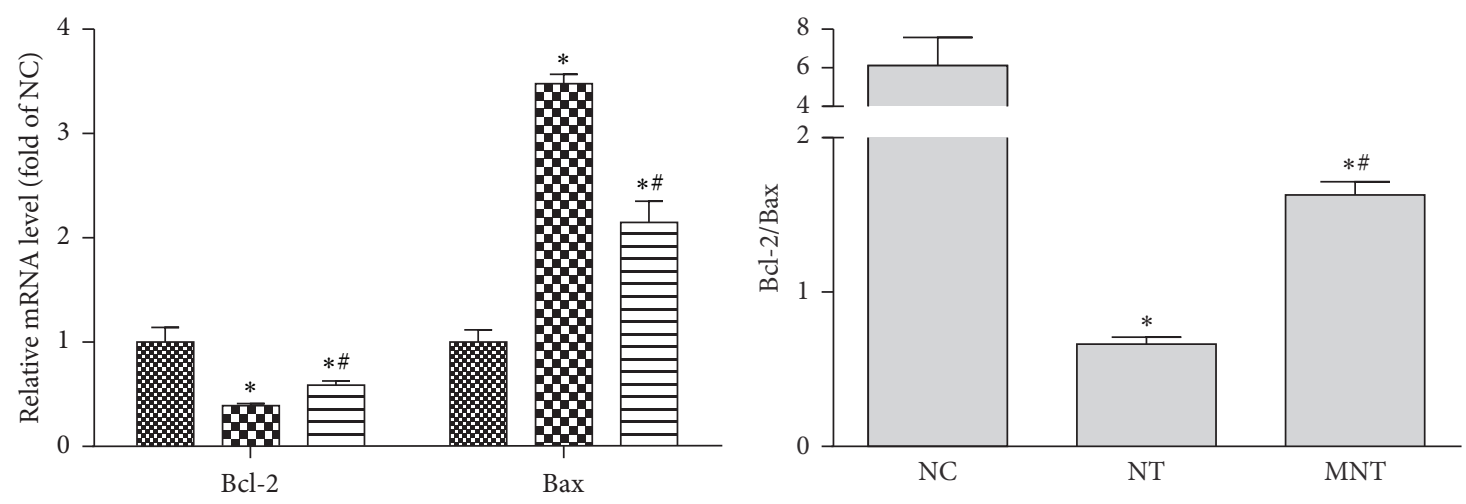

\% NC

NT

MNT

(a)

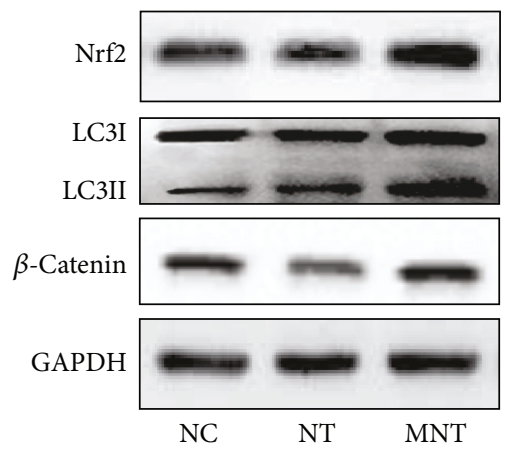

c)

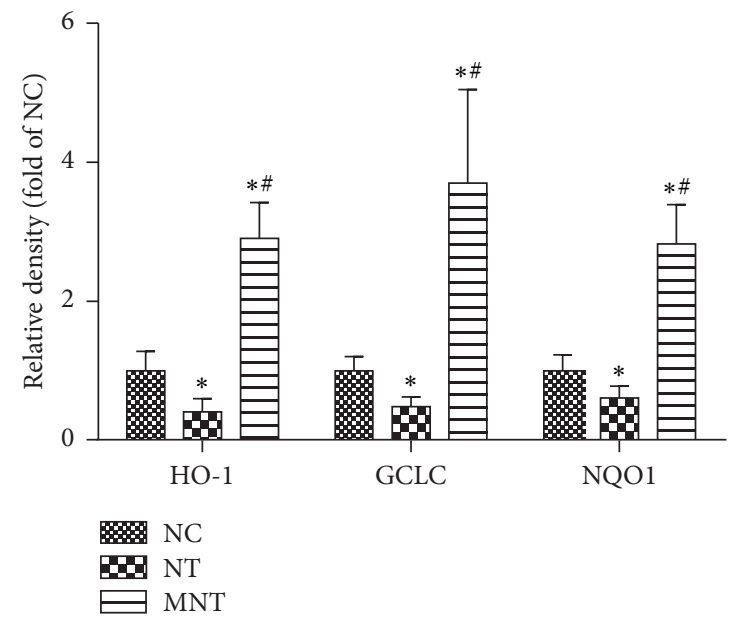

(e)

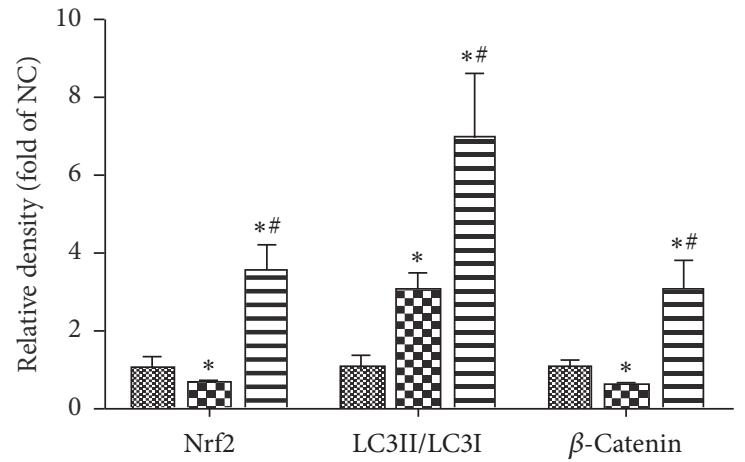

\% NC

NT

$\rightleftarrows$ MNT

(d)

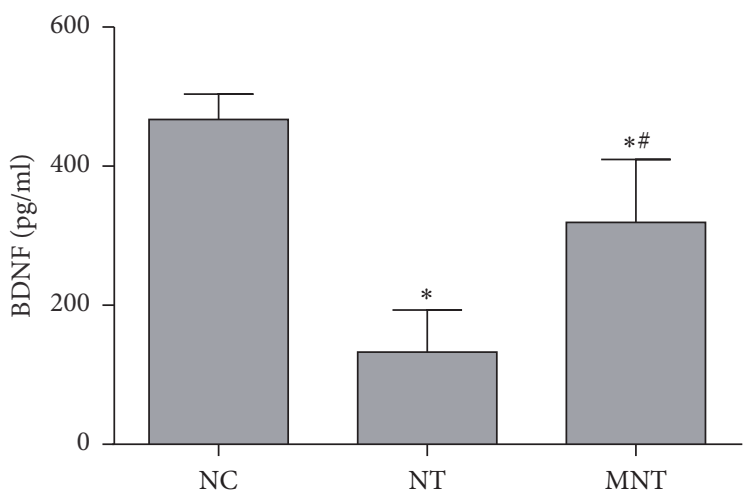

FIGURE 3: Effects of MDSC on expression of apoptosis-related factors. (a) Total RNA extracted from the cells was used for detecting the mRNA level of Bcl2 and Bax and (b) Bcl2/Bax ratio. (c) Total protein was used to observe the expression of Nrf2, LC3, and $\beta$-catenin and (d) the relative density. (e) The mRNA expression of antioxidants $(n=3)$. (f) BDNF in culture media (Day 6) was measured with ELISA kit $(n=6)$. Data was shown as mean \pm SEM, ${ }^{*} P<0.05$ versus NC group, and ${ }^{\#} P<0.05$ versus NT group. 


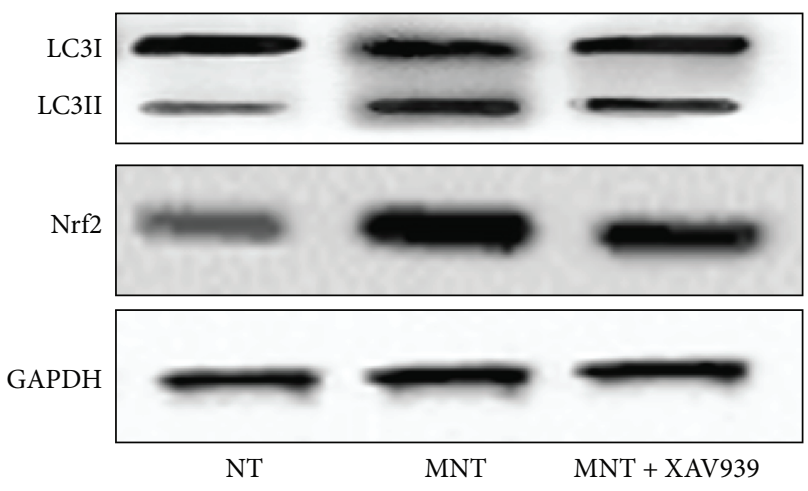

(a)

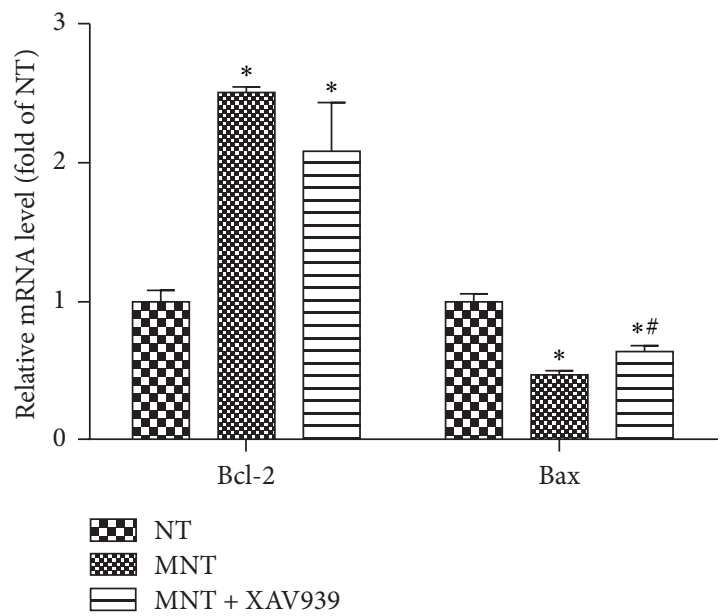

(c)

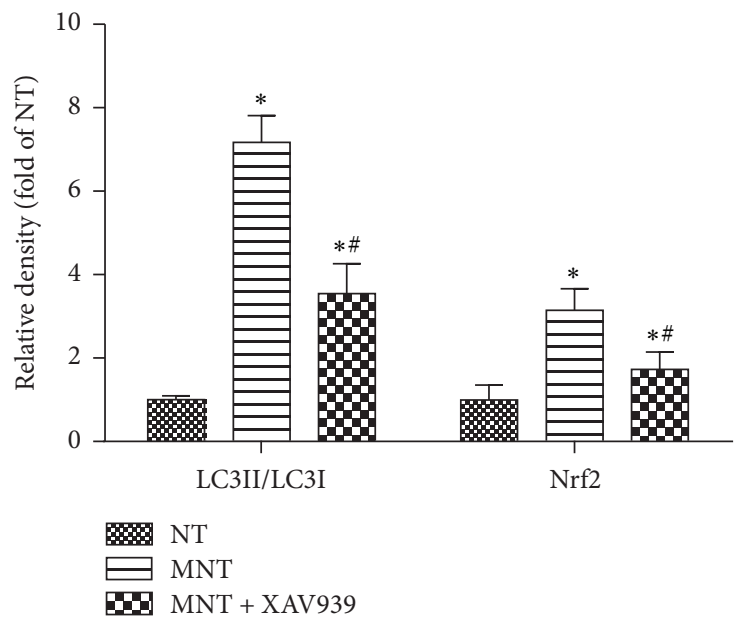

(b)

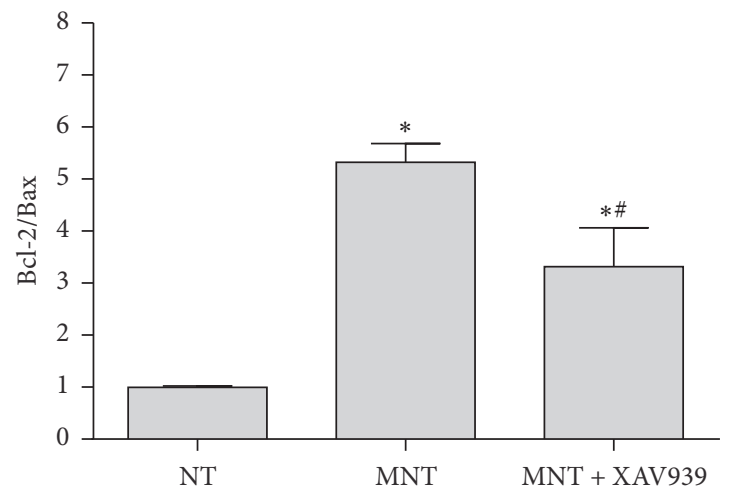

(d)

FIGURE 4: Effects of XAV939 on neuron apoptosis. To investigate the roles of $\beta$-catenin, XAV939 ( $\beta$-catenin inhibitor) was added to MNT group. (a) Total protein was used to observe the expression of Nrf2 and LC3 and (b) the relative density. (c) Total RNA extracted from the cells was used for detecting the mRNA level of Bcl2 and Bax and (d) Bcl2/Bax ratio $(n=3)$. Data was shown as mean \pm SEM, ${ }^{*} P<0.05$ versus NT group, and ${ }^{\#} P<0.05$ versus MNT group.

possibility that decreasing BDNF contributes to the injury of neurons.

\section{Discussion}

4.1. MDSCs Suppress the Progression of Apoptosis by Inhibiting Oxidative Stress and Inducing Autophagy. The secondary injury following spinal cord injury aggravates the primary lesion and exacerbates neurological impairment. Apoptosis is one of the major events in secondary injury, which affects the survivability of neurons and implanted cells [13]. During apoptosis progression, Bax is considered an important proapoptotic protein, and $\mathrm{Bcl}-2$ prevents apoptosis by downregulating Bax [14]. Furthermore, accumulating data are showing that $\mathrm{Nrf} 2$ is the crucial regulator of apoptosis by activating antioxidant cascades, such as HO-1, GCLC, and NQO1 $[15,16]$, which contribute to the suppression of apoptosis. Our data presented here support the previous reports regarding the upregulation of apoptosis (Figure 2), and we also found that MDSCs could inhibit apoptosis via the upregulation of the ratio between $\mathrm{Bcl}-2$ and $\mathrm{Bax}$ as well as antioxidants in part. Therefore, the data suggest that the protective effects of MDSCs on neuronal apoptosis, at least in part, result from the regulation of oxidative stress by upregulating Nrf2 and its downstream antioxidants (Figure 3).

Autophagy is another essential mechanism for maintaining cellular homeostasis under stress conditions. Many studies have reported that the activation of autophagy protects against pathologies $[17,18]$, and the inhibition of autophagy is associated with an increase of apoptosis, which may aggravate neuronal damage [19]. In the present study, we observed that MDSCs could promote the autophagy activation and suppress apoptosis in the MNT group compared with the NT group. Unexpectedly, we also observed the strong activation 


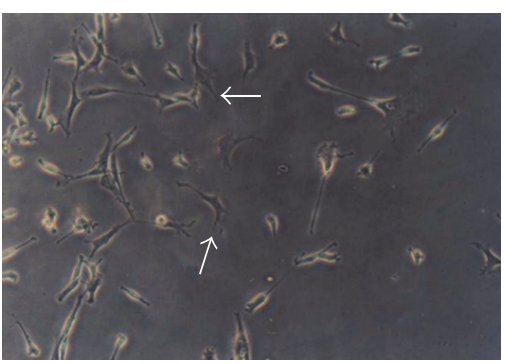

(a)

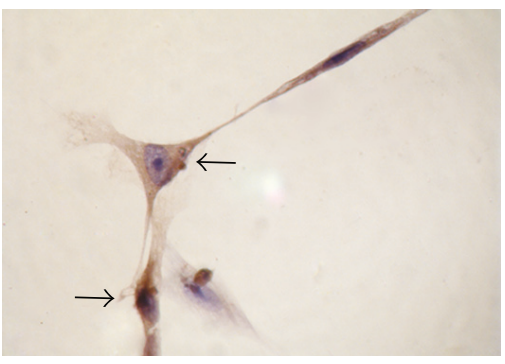

MDSCs + neurons (4th day)

(b)

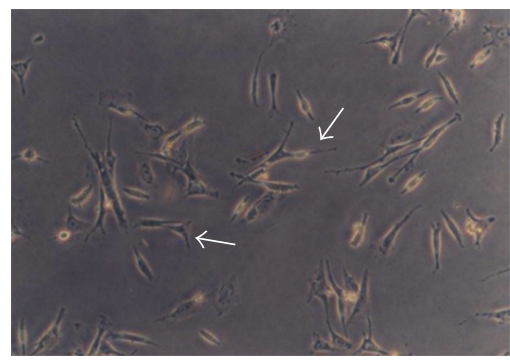

(c)

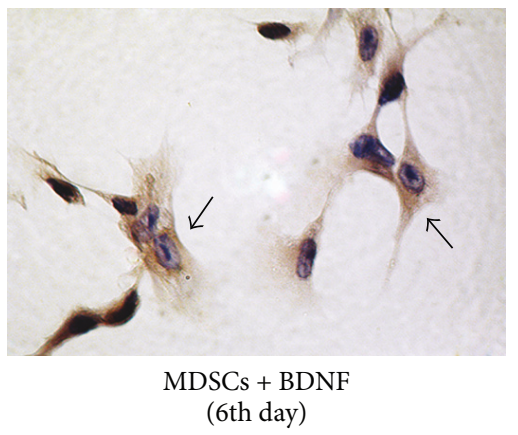

(d)

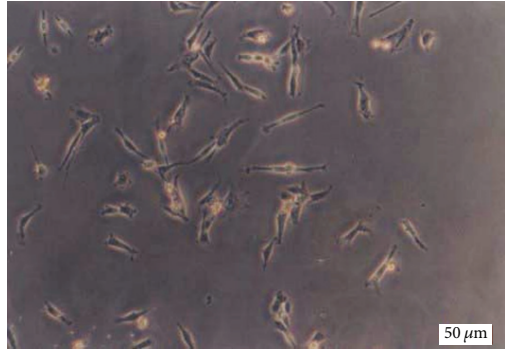

(e)

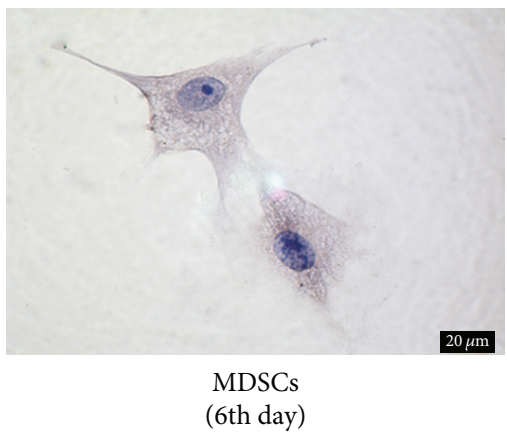

(f)
$\operatorname{BDNF}(\mathrm{pg} / \mathrm{ml})$

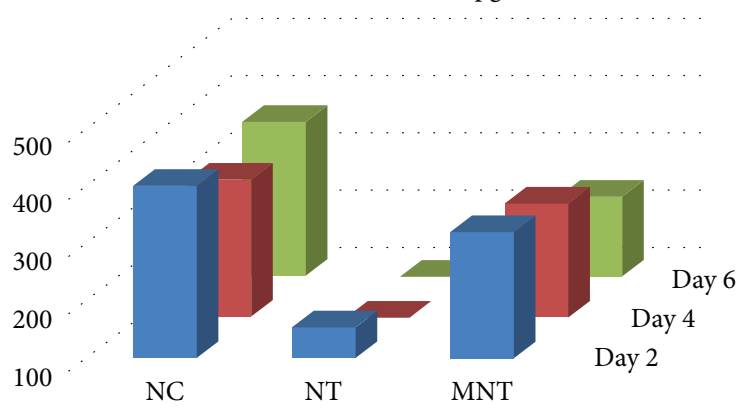

(g)

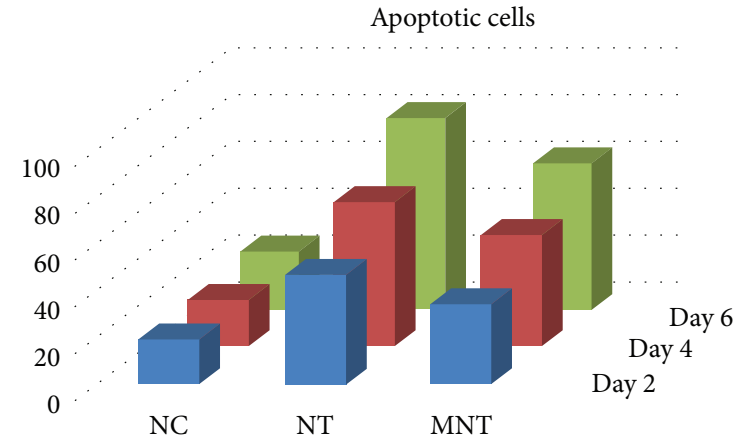

(h)

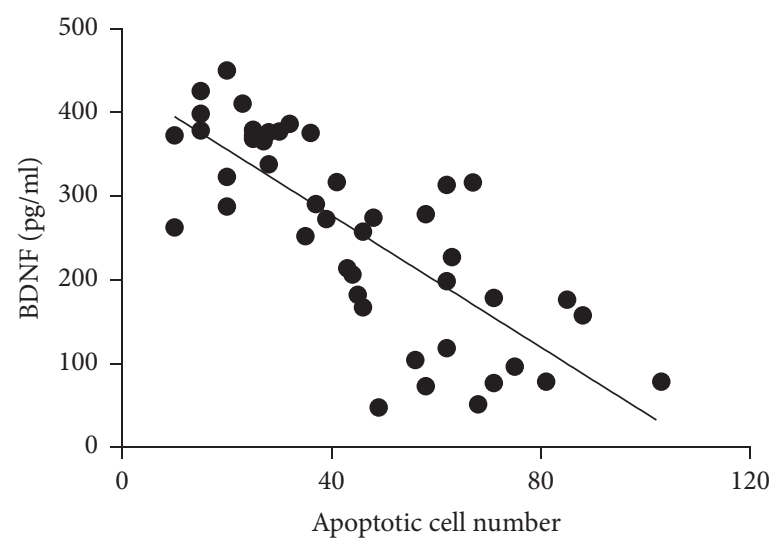

(i)

Figure 5: (a-b) The morphological changes of MDSCs in the MNT group. MDSCs were stained with NSE. (c-f) The effects of BDNF on MDSCs. MDSCs were cultured with (c, d) and without (e, f) BDNF for six days, and NSE staining was performed (d, f). Arrows $(\uparrow)$ indicate neuron-like cells $(n=3)$. Scale bar: (a, c, e) $50 \mu \mathrm{m}$; (d, e, f) $20 \mu \mathrm{m}$. (g) The level of BDNF in culture media was measured by ELISA $(n=6)$. (h) The number of apoptotic cells: after incubated with or without MDSCs, the neurons were stained with Hoechst 33258, and apoptotic cells were counted $(n=5)$. (i) The relationship between the number of apoptotic cells and the expression of BDNF. The linear regression analysis for in vitro study showed a negative correlation between BDNF and apoptosis $(R=-0.775, P<0.0001)$. 
of autophagy and apoptosis in the NT group compared with the normal group (Figure 3). Yu et al. [20] have reported that autophagy is initiated within hours to protect neurons by disposing injured components, and after few days, autophagy could induce normal cell death in the SCI model. Since the apoptosis is suppressed by MDSCs, it suggests that increased autophagy in the NT group is associated with neuron death. However, MDSCs could extend the protective effect of autophagy.

4.2. Protective Effects of BDNF Secreted by MDSCs Are Associated with $\beta$-Catenin. Recently, many studies have focused on the effects of various neurotrophic factors secreted by stem cells [21] since many scientists have proposed that the terminal differentiation of other stem cells is not a major determinant of successful tissue and function repair [22, 23]. Brain-derived neurotrophic factor (BDNF), one of the major neurotrophic factors, regulates neuronal differentiation and proliferation [24]. Some studies have provided evidence that BDNF could suppress apoptosis via the upregulating ratio of $\mathrm{Bcl}-2$ and Bax in different tissues $[25,26]$, and more recently, other reports have indicated that $\mathrm{BDNF}$ could upregulate Nrf2 [27] and autophagy [28]. The present study showed MDSCs promoted the secretion of BDNF (Figures 3 and 5), and the lower expression of BDNF was closely linked with the severity of injury (Figure 5(i)). Therefore, it is believed that MDSCs could protect the neurons from oxidative stress by BDNF.

Wnt $/ \beta$-catenin signaling defines organizing centres that orchestrate neural development, axonal guidance, cell proliferation, and neural cell survival [29]. However, the roles of $\beta$-catenin in spinal cord injury are still controversial. Several studies have reported that $\beta$-catenin is activated after SCI for promoting functional recovery [30], and other studies have shown decreased $\beta$-catenin in SCI [31]. The different results might be related to the different time point after SCI because SCI includes complex physiological and biochemical mechanisms. Various proteins are increased immediately after SCI to compensate for the injury and then decreased with the passage of time [32]. Our results also showed that MDSCs could promote $\beta$-catenin, which is downregulated in injured neurons. The inhibition of $\beta$-catenin results in reduced autophagy, antioxidants, and increased apoptosis. The results are consistent with previous reports that indicated the roles of $\beta$-catenin on apoptosis and autophagy. It is reported that $\beta$-catenin could inhibit apoptosis via regulating CYP21A2 [33], and the alteration of $\beta$-catenin is closely associated with autophagy in SCI rats [31]. Interestingly, BDNF has been reported to regulate $\beta$-catenin [34]. Therefore, protective effects of MDSCs on injured neurons, at least in part, result from the increased presence of $\beta$-catenin, which is regulated by BDNF.

4.3. BDNF Could Stimulate Neuron-Like Cell Differentiation. In an experiment to further verify the roles of MDSCs under a stress microenvironment, we observed a large number of NSE-positive neuron-like cells in the MNT group (Figures $4(\mathrm{a})-4(\mathrm{~d}))$. We also found that the presence of exogenous
BDNF stimulated the transformation of MDSCs into NSEneuron-like cells. Increasing studies have revealed that various stem cells also can be induced into neuron-like cells by treatment with $\operatorname{BDNF}[35,36]$. Hence, we speculate that MDSCs may primarily stimulate neurons to secrete BDNF, which could eventually affect the terminal differentiation of MDSCs into neuron-like cells. Neuron-like cells are also believed to replace injured neurons and exert neuronal-like roles under oxidative conditions. However, further studies need to investigate the exact roles of neuron-like cells in SCI rats.

\section{Conclusions}

Our findings showed that the decreased apoptosis rate by the MDSCs is related to the increased BDNF. BDNF might not only upregulate autophagy but also downregulate oxidative stress. Moreover, BDNF promotes the transition of MDSCs into neuron-like cells, which could replace injured neurons. In the present study, we also found $\beta$-catenin is involved with the protective effects of MDSCs by inducing antioxidants and autophagy. These results suggest that MDSCs could be potential therapeutic approaches for the treatment of spinal cord injuries.

\section{Conflicts of Interest}

The authors declare that there are no conflicts of interest regarding the publication of this article.

\section{Authors' Contributions}

Donghe Han and Shurui Chen contributed equally to this work.

\section{Acknowledgments}

This project was financially supported by the Project for NSFC [81171799, 81300534, and 81300620] and Program for Liaoning Innovative Research Team [2008T113].

\section{References}

[1] C. Penas, M.-S. Guzmán, E. Verdú, J. Forés, X. Navarro, and C. Casas, "Spinal cord injury induces endoplasmic reticulum stress with different cell-type dependent response," Journal of Neurochemistry, vol. 102, no. 4, pp. 1242-1255, 2007.

[2] Y. J. Moon, J. Y. Lee, M. S. Oh et al., "Inhibition of inflammation and oxidative stress by Angelica dahuricae radix extract decreases apoptotic cell death and improves functional recovery after spinal cord injury," Journal of Neuroscience Research, vol. 90, no. 1, pp. 243-256, 2012.

[3] J. Zhou, P. Lu, H. Ren et al., " $17 \beta$-estradiol protects human eyelid-derived adipose stem cells against cytotoxicity and increases transplanted cell survival in spinal cord injury," Journal of Cellular and Molecular Medicine, vol. 18, no. 2, pp. 326-343, 2014.

[4] C. Gu, H. Li, C. Wang et al., "Bone marrow mesenchymal stem cells decrease CHOP expression and neuronal apoptosis after 
spinal cord injury," Neuroscience Letters, vol. 636, pp. 282-289, 2017.

[5] J. F. Bonner and O. Steward, "Repair of spinal cord injury with neuronal relays: from fetal grafts to neural stem cells," Brain Research, vol. 1619, pp. 115-123, 2015.

[6] A. Usas, J. Maciulaitis, R. Maciulaitis, N. Jakuboniene, A. Milasius, and J. Huard, "Skeletal muscle-derived stem cells: implications for cell-mediated therapies," Medicina (Kaunas), vol. 47, pp. 469-479, 2011.

[7] J. S. Oh, K. N. Kim, S. S. An et al., "Cotransplantation of mouse neural stem cells (mNSCs) with adipose tissue-derived mesenchymal stem cells improves mNSC survival in a rat spinal cord injury model," Cell Transplantation, vol. 20, no. 6, pp. 837849, 2011.

[8] S. B. Jazayeri, S. Beygi, F. Shokraneh, E. M. Hagen, and V. Rahimi-Movaghar, "Incidence of traumatic spinal cord injury worldwide: a systematic review," European Spine Journal, vol. 24, no. 5, pp. 905-918, 2015.

[9] T. J. Burkholder, B. Fingado, S. Baron, and R. L. Lieber, "Relationship between muscle fiber types and sizes and muscle architectural properties in the mouse hindlimb," Journal of Morphology, vol. 221, no. 2, pp. 177-190, 1994.

[10] J. Y. Lee, Z. Qu-Petersen, B. Cao et al., "Clonal isolation of muscle-derived cells capable of enhancing muscle regeneration and bone healing," Journal of Cell Biology, vol. 150, no. 5, pp. 1085-1100, 2000.

[11] L. Danisovic, I. Varga, S. Polak, M. Ulicna, D. Bohmer, and J. Vojtassak, "Morphology of in vitro expanded human musclederived stem cells," Biomedical Papers of the Medical Faculty of the University Palacky, Olomouc, Czech Republic, vol. 152, no. 2, pp. 235-238, 2008.

[12] J. Tsao, D. A. Vernet, R. Gelfand et al., "Myostatin genetic inactivation inhibits myogenesis by muscle-derived stem cells in vitro but not when implanted in the mdx mouse muscle," Stem Cell Research and Therapy, vol. 4, no. 1, article 4, 2013.

[13] J. Mateos, A. De la Fuente, I. Lesende-Rodriguez, P. FernándezPernas, M. C. Arufe, and F. J. Blanco, "Lamin A deregulation in human mesenchymal stem cells promotes an impairment in their chondrogenic potential and imbalance in their response to oxidative stress," Stem Cell Research, vol. 11, no. 3, pp. 1137-1148, 2013.

[14] X.-F. Huang, S.-K. Luo, J. Xu et al., "Aurora kinase inhibitory VX-680 increases $\mathrm{Bax} / \mathrm{Bcl}-2$ ratio and induces apoptosis in Aurora-A-high acute myeloid leukemia," Blood, vol. 111, no. 5, pp. 2854-2865, 2008.

[15] J. W. Kaspar, S. K. Niture, and A. K. Jaiswal, "Nrf2:INrf2 (Keap1) signaling in oxidative stress," Free Radical Biology and Medicine, vol. 47, no. 9, pp. 1304-1309, 2009.

[16] Z. Zhai, S. E. Gomez-Mejiba, M. S. Gimenez et al., "Free radical-operated proteotoxic stress in macrophages primed with lipopolysaccharide," Free Radical Biology and Medicine, vol. 53, no. 1, pp. 172-181, 2012.

[17] L. Zhang, P. Tang, H. Hou et al., "Autophagy reduces neuronal damage and promotes locomotor recovery via inhibition of apoptosis after spinal cord injury in rats," Molecular Neurobiology, vol. 49, no. 1, pp. 276-287, 2014.

[18] D. Zhang, B. Tang, X. Xie, Y.-F. Xiao, S.-M. Yang, and J.-W. Zhang, "The interplay between DNA repair and autophagy in cancer therapy," Cancer Biology and Therapy, vol. 16, no. 7, pp. 1005-1013, 2015.
[19] T. Hara, K. Nakamura, M. Matsui et al., "Suppression of basal autophagy in neural cells causes neurodegenerative disease in mice," Nature, vol. 441, no. 7095, pp. 885-889, 2006.

[20] H. Yu, L. Li, R. Liu et al., "Autophagy in long propriospinal neurons is activated after spinal cord injury in adult rats," Neuroscience Letters, vol. 634, pp. 138-145, 2016.

[21] M. Sasaki, C. Radtke, A. M. Tan et al., "BDNF-hypersecreting human mesenchymal stem cells promote functional recovery, axonal sprouting, and protection of corticospinal neurons after spinal cord injury," The Journal of Neuroscience, vol. 29, no. 47, pp. 14932-14941, 2009.

[22] J. Chen, H.-C. Park, F. Addabbo et al., "Kidney-derived mesenchymal stem cells contribute to vasculogenesis, angiogenesis and endothelial repair," Kidney International, vol. 74, no. 7, pp. 879-889, 2008.

[23] L. B. Balsam, A. J. Wagers, J. L. Christensen, T. Kofidis, I. L. Weissmann, and R. C. Robbins, "Haematopoietic stem cells adopt mature haematopoietic fates in ischaemic myocardium," Nature, vol. 428, no. 6983, pp. 668-673, 2004.

[24] C. Anthony Altar, N. Cai, T. Bliven et al., "Anterograde transport of brain-derived neurotrophic factor and its role in the brain," Nature, vol. 389, no. 6653, pp. 856-860, 1997.

[25] L. Hwang, I.-Y. Choi, S.-E. Kim et al., "Dexmedetomidine ameliorates intracerebral hemorrhage-induced memory impairment by inhibiting apoptosis and enhancing brain-derived neurotrophic factor expression in the rat hippocampus," International Journal of Molecular Medicine, vol. 31, no. 5, pp. 10471056, 2013.

[26] W.-R. Schabitz, C. Sommer, W. Zoder et al., "Intravenous brain-derived neurotrophic factor reduces infarct size and counterregulates Bax and Bcl-2 expression after temporary focal cerebral ischemia," Stroke, vol. 31, no. 9, pp. 2212-2217, 2000.

[27] J. S. Allard, E. J. Perez, K. Fukui, P. Carpenter, D. K. Ingram, and R. D. Cabo, "Prolonged metformin treatment leads to reduced transcription of Nrf2 and neurotrophic factors without cognitive impairment in older C57BL/6J mice," Behavioural Brain Research, vol. 301, pp. 1-9, 2016.

[28] K. Gao, G. Wang, Y. Wang et al., "Neuroprotective effect of simvastatin via inducing the autophagy on spinal cord injury in the rat model," BioMed Research International, vol. 2015, Article ID 260161, 9 pages, 2015.

[29] S. Zhang, J. Li, R. Lea, K. Vleminckx, and E. Amaya, "Fezf2 promotes neuronal differentiation through localised activation of Wnt/ $\beta$-catenin signalling during forebrain development," Development, vol. 141, no. 24, pp. 4794-4805, 2014.

[30] L. Sun, J. Pan, Y. Peng et al., "Anabolic steroids reduce spinal cord injury-related bone loss in rats associated with increased Wnt signaling," Journal of Spinal Cord Medicine, vol. 36, no. 6, pp. 616-622, 2013.

[31] G. S. Miranpuri, D. T. Schomberg, B. Alrfaei et al., "Role of matrix metalloproteinases 2 in spinal cord injury-induced neuropathic pain," Annals of Neurosciences, vol. 23, no. 1, pp. 2532, 2016.

[32] J. Zhang, G. Feng, G. Bao et al., "Nuclear translocation of PKM2 modulates astrocyte proliferation via p27 and $\beta$-catenin pathway after spinal cord injury," Cell Cycle, vol. 14, no. 16, pp. 2609-2618, 2015.

[33] L. F. Leal, A. C. Bueno, D. C. Gomes, R. Abduch, M. de Castro, and S. R. Antonini, "Inhibition of the Tcf/beta-catenin complex increases apoptosis and impairs adrenocortical tumor cell proliferation and adrenal steroidogenesis," Oncotarget, vol. 6, no. 40, pp. 43016-43032, 2015. 
[34] L. Z. Xu, D. F. Xu, Y. Han et al., "BDNF-GSK-3beta-beta-catenin pathway in the $\mathrm{mPFC}$ is involved in antidepressant-like effects of morinda officinalis oligosaccharides in rats," International Journal of Neuropsychopharmacology, vol. 20, no. 1, pp. 83-93, 2017.

[35] C. Clow and B. J. Jasmin, "Brain-derived neurotrophic factor regulates satellite cell differentiation and skeltal muscle regeneration," Molecular Biology of the Cell, vol. 21, no. 13, pp. 21822190, 2010.

[36] N. D. Leipzig, C. Xu, T. Zahir, and M. S. Shoichet, "Functional immobilization of interferon-gamma induces neuronal differentiation of neural stem cells," Journal of Biomedical Materials Research-Part A, vol. 93, no. 2, pp. 625-633, 2010. 

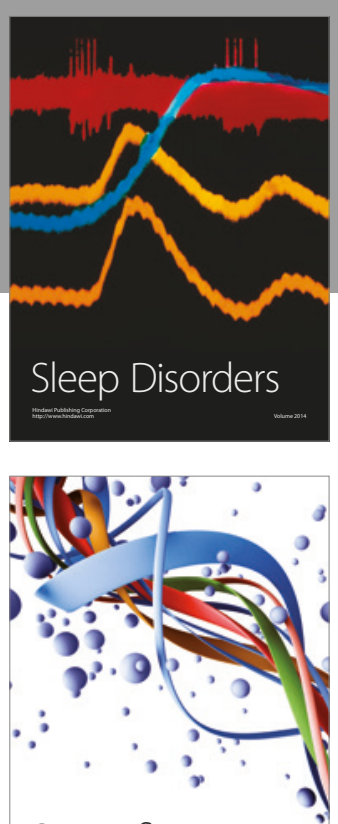

Scientifica
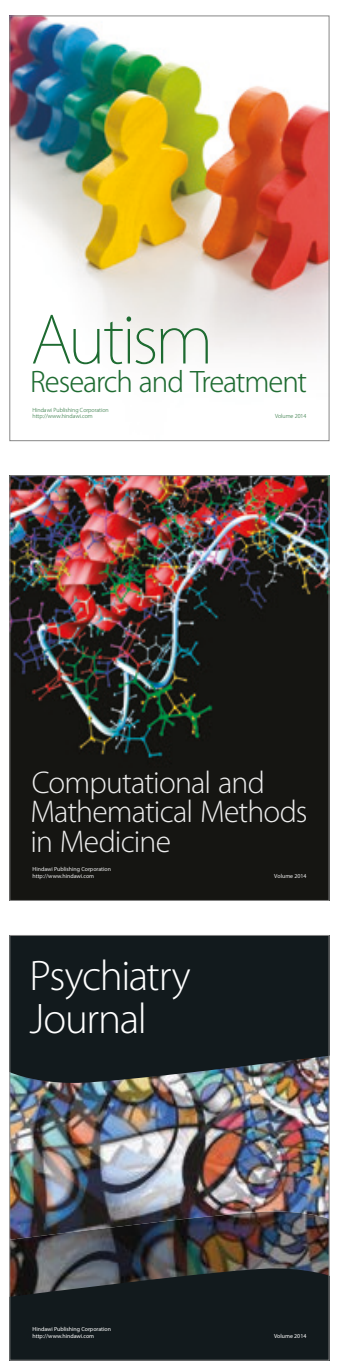
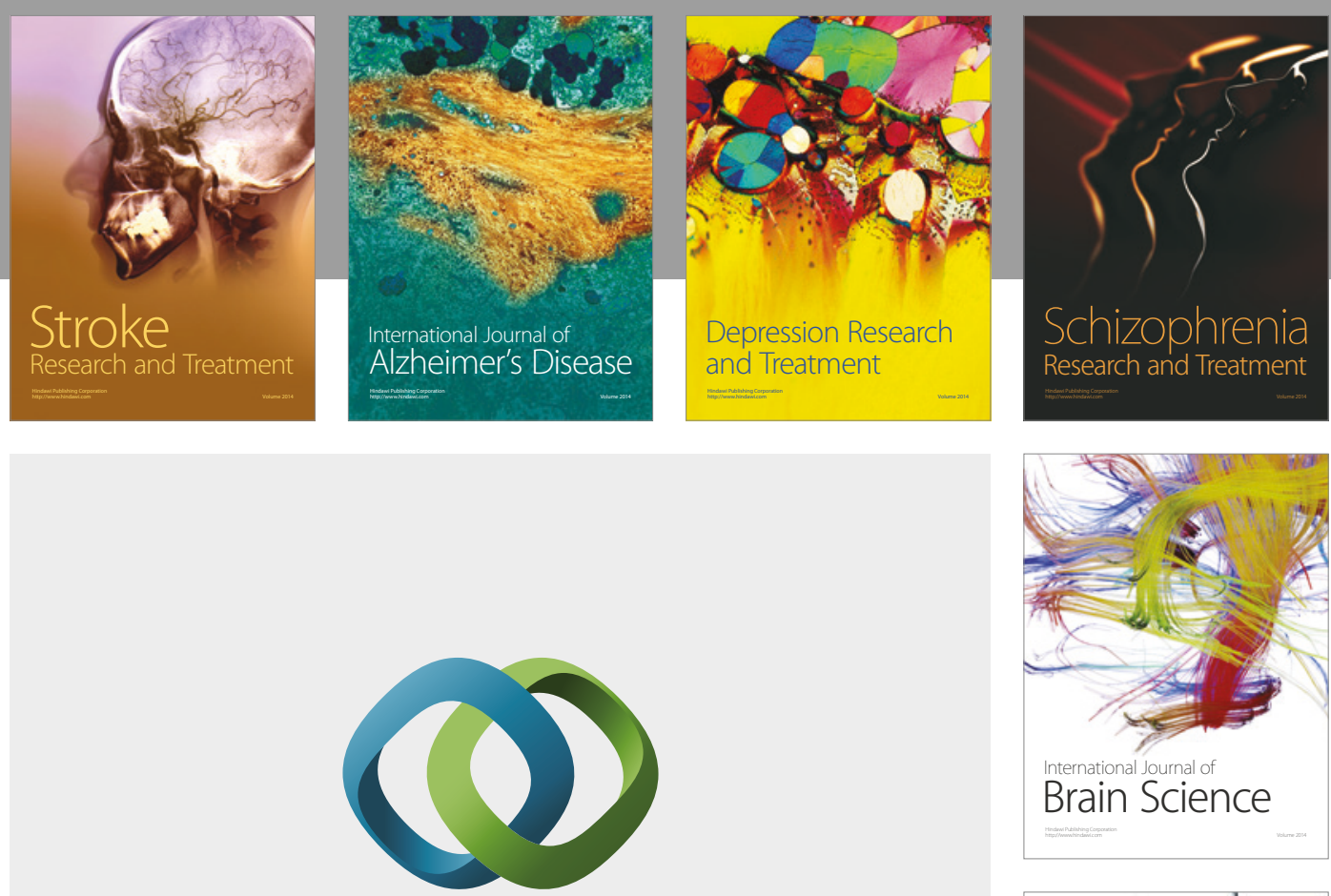

\section{Hindawi}

Submit your manuscripts at

https://www.hindawi.com
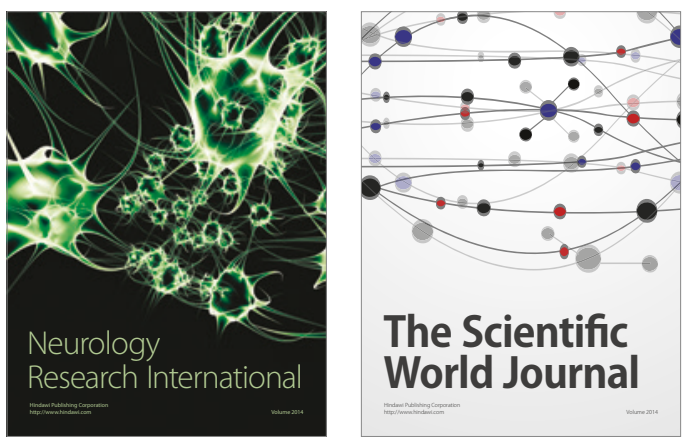

The Scientific World Journal

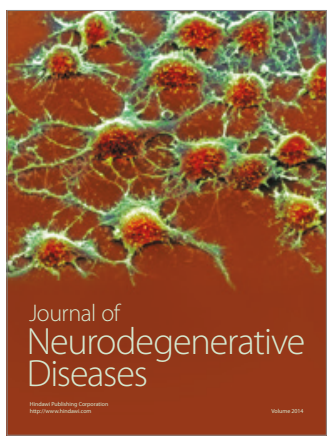

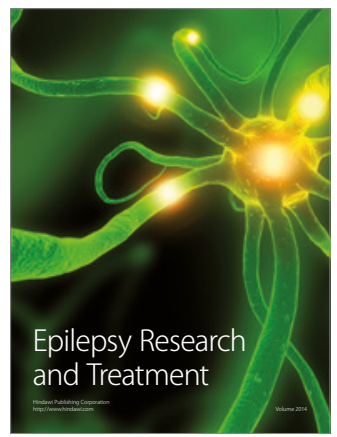

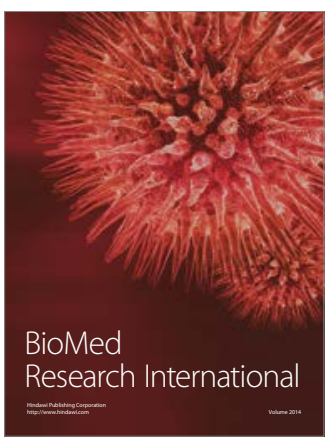

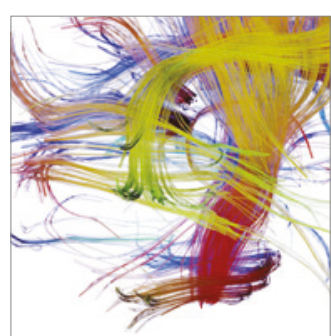

Brain Science

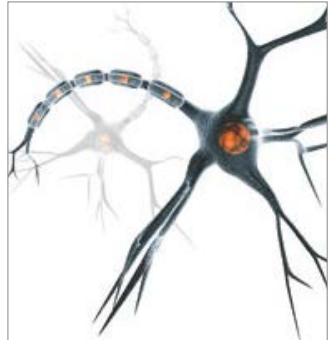

Neural Plasticity
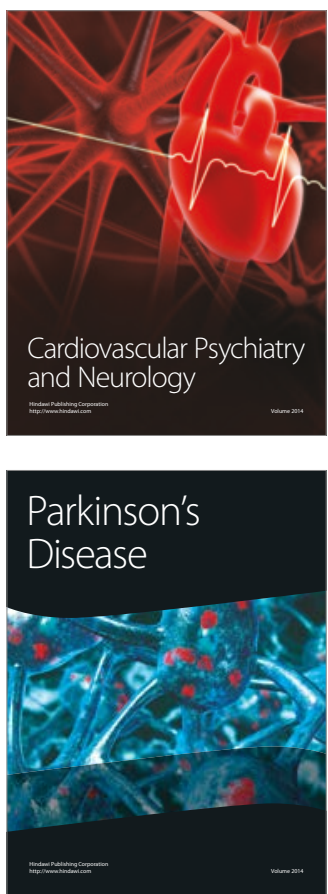